\title{
Automatization of Optimal Routing for Multimodal Passenger Transportations in Irkutsk Region
}

\author{
Krol Nikita \\ Irkutsk State Transport University \\ 15 Chernishevsky St., Irkutsk, Russia \\ krolnikitav@mail.ru
}

\author{
Poletaev Alexander \\ Irkutsk National Research Technical University \\ 83 Lermontov St., Irkutsk, Russia. \\ sardaukar9@yandex.ru
}

\author{
Upyr Roman \\ Irkutsk State Transport University \\ 15 Chernishevsky St., Irkutsk, Russia \\ upyr.roman@gmail.com
}

\begin{abstract}
The article presents an algorithm of forming dynamic table of routs for multimodal passenger transportations. The algorithm selects the optimum route using one of criteria: fare, travelling time, comfort level. It is shown that the proposed method can find routs with minimum number of transfers. The algorithm is realized in the graphical language of programming LabVIEW. It has been tested for Irkutsk region touristic routs.
\end{abstract}

Keywords - routing, algorithms of route selecting, multimodal transportations, automation of transportations control, LabVIEW, combined transports

\section{INTRODUCTION}

Organization of multimodal transportations is one of passenger transport systems directions that is actively being developed. Building wide multimodal networks is perspective not only in the regional level but also needed for urban environment of megalopolises $[1,2]$.

Different model variants for combined transportations in [3] use for the optimal route selecting a serial search of all transition ways with comparing of cost and journey time. It is shown in review [4] how different vehicles could be classified for combined passenger transports. Besides that, the authors suggest that planning of routes should be separate for transportations of passengers and their massive luggage.

Discussed in the article [5] concept of routing for autotransport tourists proposes to use the following criteria: route distances, transition time, quality of service, safety level and so on. At the end all these particular factors are being converted into a generalized preference scale.

But in our opinion, the most important parameters for passengers are total cost, journey time and comfort level. Buisness trips can be characterized by urgency so it is important to shorten time loss. People who are used to travelling would have a desire to save money. Someone would prefer to enjoy their journey with maximum conveniences.

\section{Problem Definition}

For organizing of goods transportation routing there are a lot of approaches and algorithms [6]. The main attention there is devoted to principles of loop routes constructing with minimization of total route length. Also different genetic algorithms are becoming more popular [7, 8], their operation is based on random search principle. The issue of optimization for passenger transportation can be related to the problem class for finding the shortest distance between a couple of cities [9].

Network protocols for data routing or solving the transport task are commonly involve Dijkstra's algorithm [10]. In this method, finding the way with minimum weighted length is determined via serial selecting the most of route map points. However for multimodal transportations such approach is not very efficient. It will require making an exhaustive search, close alternative routes to the shortest one are not taken into account at the end. In addition, the algorithm does not consider multiple layers of routing maps.

The figure 1 show some variants of touristic travelling lines for different transportation modes in Irkutsk Region and Respublika Buryatiya [11]. The identification numbers of points on the map are explicated in the Table I. Multimodal transportations for these regions include railroads, motor vehicles (regular bases, taxi), airlines and water transport. Each segment of the map can combine several variants of transition not only for different modes but also for several transport companies.

As it can be seen from the figure 1, even relatively low number of touristic places produces a wide transportation net in case of multimodal system. In order not to complicate the example, there are only the most popular tourist routes on the map. The places for visiting can be also different for summer and winter touristic seasons. 


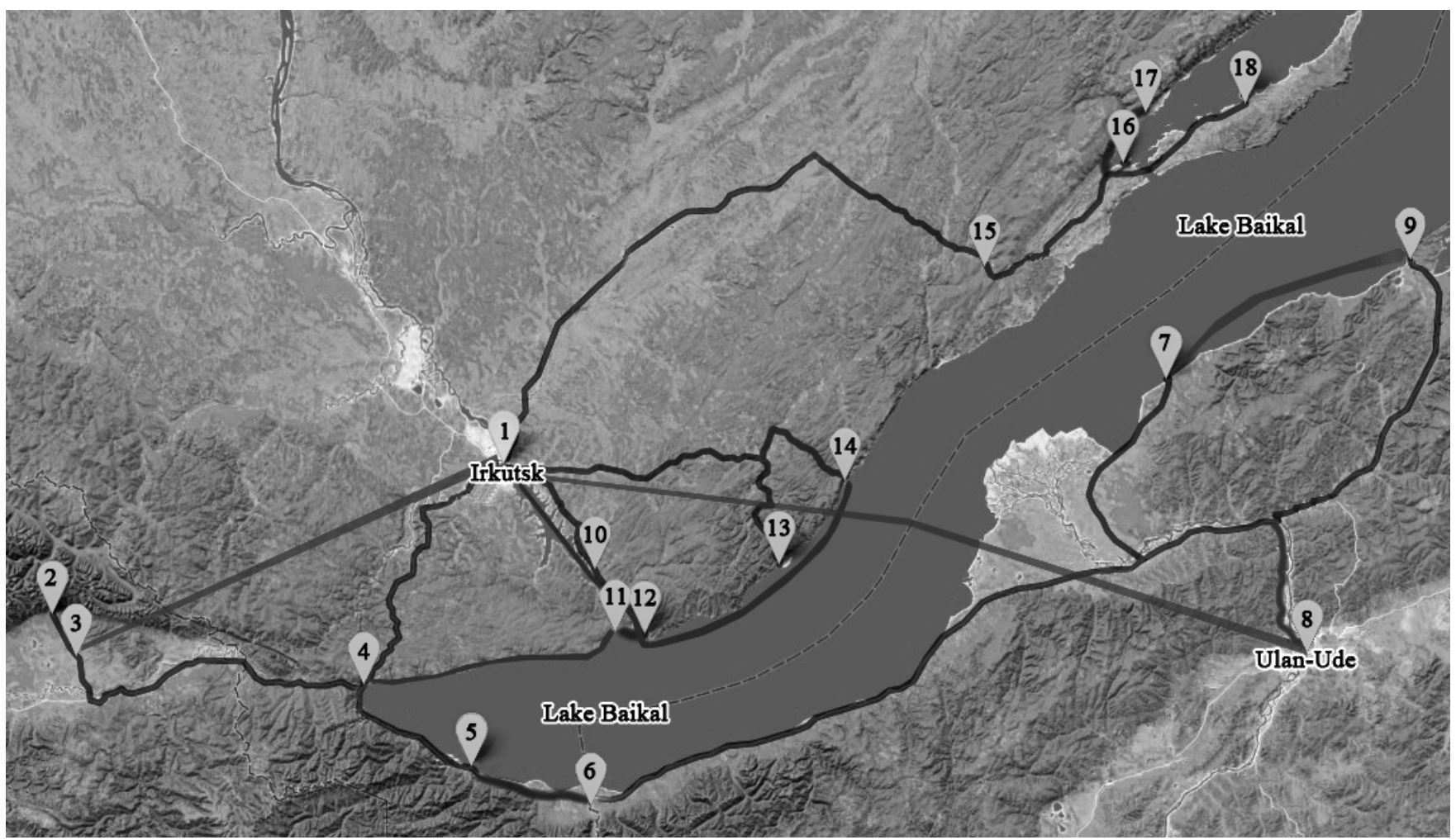

Fig. 1. A map of popular tourisric routes in Irkutsk Region.

Self-reliant planning of transitions is quite hard for passengers especially when they have time limitations. Even if there is an integrated data base providing with information about all transport mode schedules, a passenger will be not capable to find the most suitable and available route.

Thereby, there is a requirement of automated forming a table of all transition combinations of travelling lines, and selecting the optimum route with taking into account passenger preferences and multimodal character of transportations.

The aim of the article is to design an algorithm of selecting the optimal route and verify its operating for touristic routes in Irkutsk Region.

TABLE I. LIST OF POPULAR TOURISIC PACES SHOWN IN FIGURE 1

\begin{tabular}{|c|c|c|c|}
\hline № & Place & № & Place \\
\hline 1 & Irkutsk & 10 & Talci \\
\hline 2 & Arshan & 11 & Baikal Harbor \\
\hline 3 & Tunkinskaya Valley & 12 & Listvyanka \\
\hline 4 & Slyudyanka & 13 & Bolshoye Goloustnoye \\
\hline 5 & Baikalsk & 14 & Peschanaya Bay \\
\hline 6 & Vydrino & 15 & Tazheransky Steppes Caves \\
\hline 7 & Enkhaluk & 16 & Maloe More \\
\hline 8 & Ulan-Ude & 17 & Sarminskoe Ravine \\
\hline 9 & Gremyachinsk & 18 & Olkhon \\
\hline
\end{tabular}

\section{ALGORITHM OF DYNAMIC ROUTING}

The proposed method is based on cyclic forming of route table with accumulating of transitions. A block diagram of using operations is shown in the figure 2 .

On the first step, the information about segments of the route map is loaded from a txt-file. A user inputs a point of departure and a place of arrival via choosing variants from automatically formed list of available points. Then one of selecting criteria is marked. It can be minimum cost of journey, shortest time or maximum comfort level.

Next step begins with making a list of segments and forming an adjacency and incidence matrices. In cells of the adjacency matrix are written information about all variants of transition between neighbor points. The incidence matrix only contain "NaN" and " 1 " values.

After that that we need to build dynamically a route table. Dynamic forming means that all routes are composed step-bystep in parallel via adding a new transition every loop iteration.

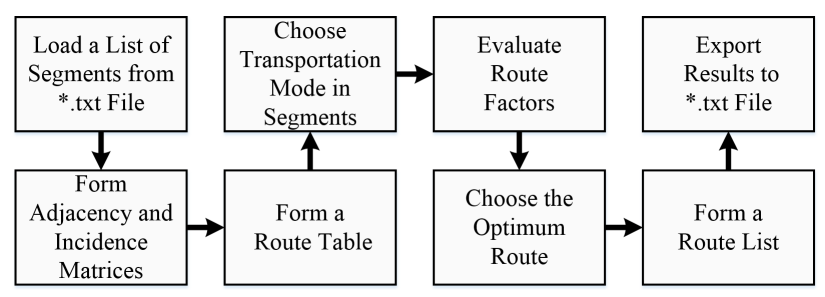

Fig. 2. Block diagram of the algorithm. 

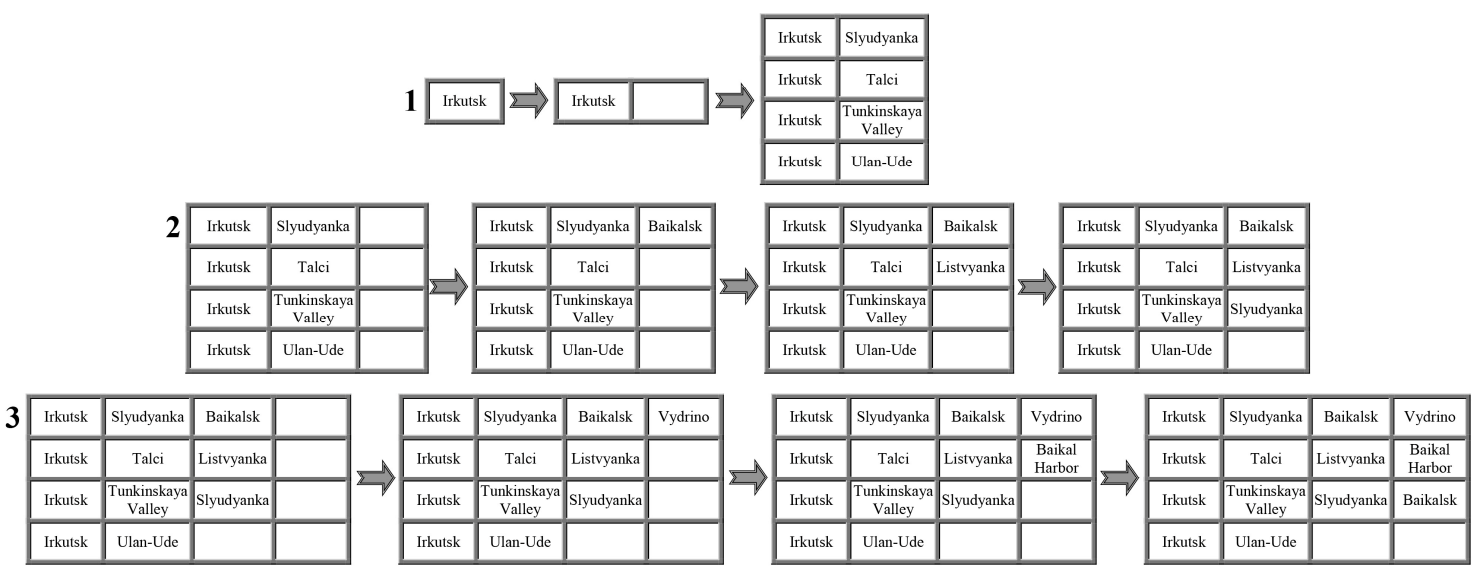

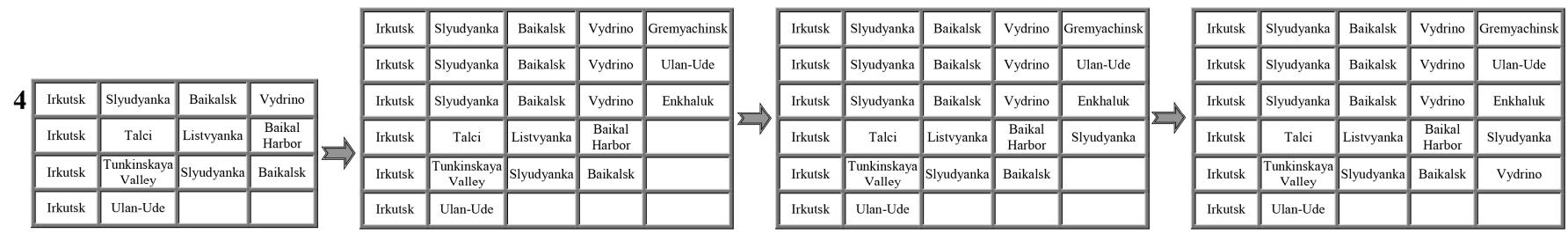

Fig. 3. Diagram of dynamic forming of a route table. Numbers indicate couting of loop iterations.

Forming routes are filled in the table in rows. During each iteration, the algorithm adds new columns and checks if a route in the row is finished or not. A route becomes pointless if it contains transit points more than one time.

An example of step-by-step finding variants of transition from Irkutsk to Ulan-Ude is presented in figure 3 . The procedure reminds of process of a tree roots system growth and splitting. For this reason, graphs representing such routing tables are often called trees. Succession of operations:

1. Initializing. At first, it is created a string table with the only one cell. Then the departure point is inserted into the cell.

2. Annual growth of roots (outer loop). It is added an empty column on the right of the table. After that all rows are checked in the inner loop:

- $\quad$ All neighbor points are found for the last non-empty element of an analyzing row. The current row is duplicated and one of found adjacent points is inserted at the end of every line copy. In our analogy, it is equivalent of root spitting.

- If the last non-empty element is the destination point, then the last column of current row is left empty.

3. Dying of growing upward roots. It is checked weather transition points are repeated in rows. If a route is looped then the whole row is removed from the table.

4. Checking the limits of roots' spreading. Here we need to evaluate a threshold which indicates a relative number of rows with accomplished routes. If this ratio exceeds a certain percent, the outer loop is stopped. Otherwise, we should go to the second step and repeat one more cycle of the table enlarging.
At the end of the procedure we get a table that contain all possible transit points for transportation from the departure place to the chosen arrival point. The final route table for the example is in figure 4 . If we set a $14 \%$ threshold of filling then there will be only one variant with minimum amount of transitions: airline Irkutsk - Ulan-Ude.

The next step of the algorithm shown in the figure 2 is choosing a way of transition for each segment of formed routes. At first, corresponding cells from the adjacent matrix are entirely copied into a new table of transitions. Then information in every cell is sorted according to the chosen criteria.

If the main priority is minimization of cost, we leave only data for low-cost fare. After that, the residual variants are sorted with ascending of journey time. We should leave the shortest ones. And, at last, if there are still several modes, we exclude variants with low level of comfort. In otherk words, the order of priorities is "cost - time - comfort". Another possible way of sequent selecting is excluding sorting "time cost - comfort", or "comfort - time - cost".

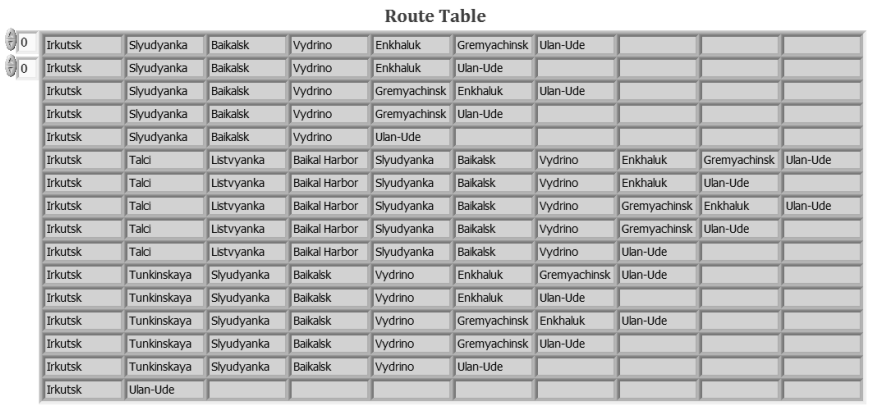

Fig. 4. An example of finished routing table for passenger transportations Irkutsk - Ulan-Ude 
When a transport mode is chosen in every segment, the algorithm evaluates total costs, general time and mean level of comfort for each row in the route table. The priorities and the procedure of route selecting is analogous. Eventually a route list contain information about transitions most corresponding to passenger's requirements.

If there are routs with the same set of characteristics, alternative variates are also included into the route list. The results are displayed on the screen and exported to a txt-file.

\section{Programming OF THE ALGoritm}

For automatic execution of the operations according to the algorithm we have created a program in graphical medium LabVIEW. Advantages of this language of programming are fast developing of applications, high functionality, and convenient visual indication and control.

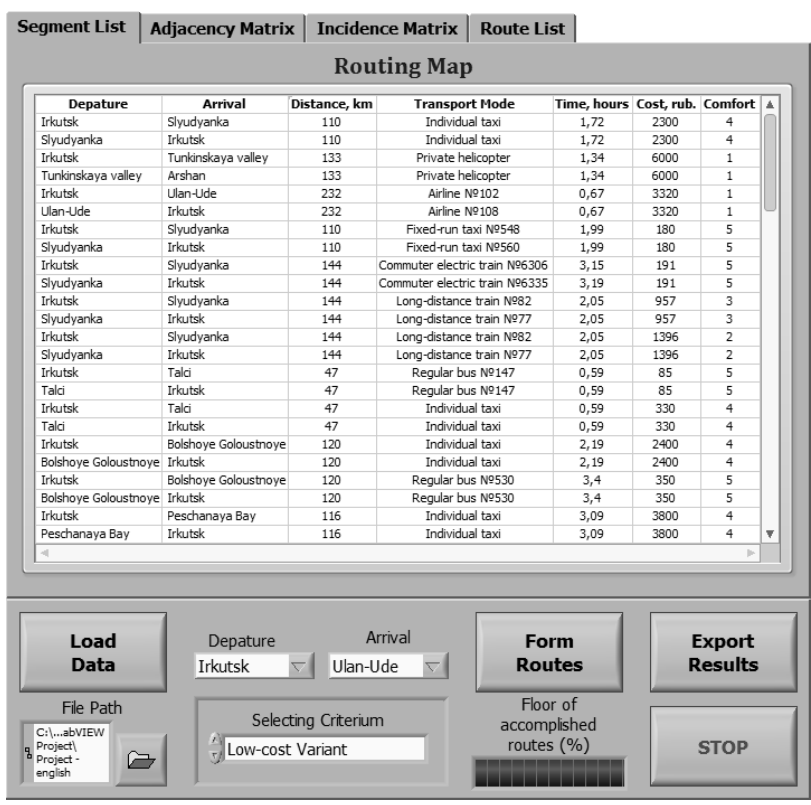

Figure 5 presents the front panel of user interface and block diagram of code in the main program. On the first page of the menu you can see a list of segments for Irkutsk Region route map. The following two pages indicate the adjacency and incidence matrices.

The last page of the menu show the route table and the route list with the results of the program run. There is only one route conforming to the selected requirement of minimum cost. It is the path "Irkutsk - Slyudyanka - Baikalsk - Vydrino - Ulan-Ude". For test input data the total cost is 990 roubles, journey time is 7.46 hours. Admittedly, it is the cheapest variant among 15 other routes.

The program is based on module programming principle. The operations from figure 2 are realized in subprograms (modules). Pushing of virtual buttons is processed in the Event Structure.

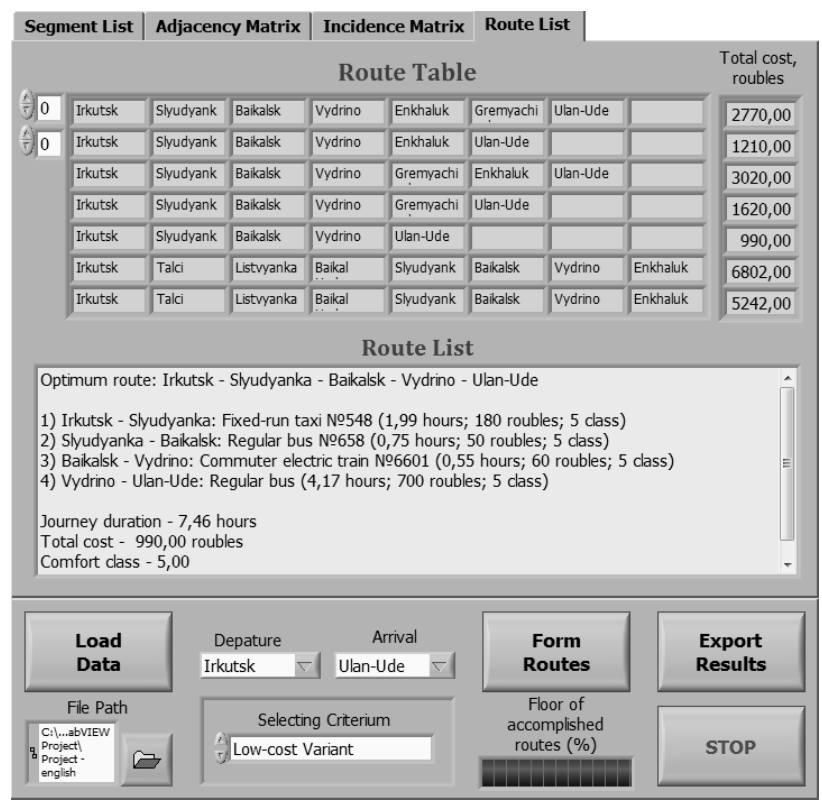

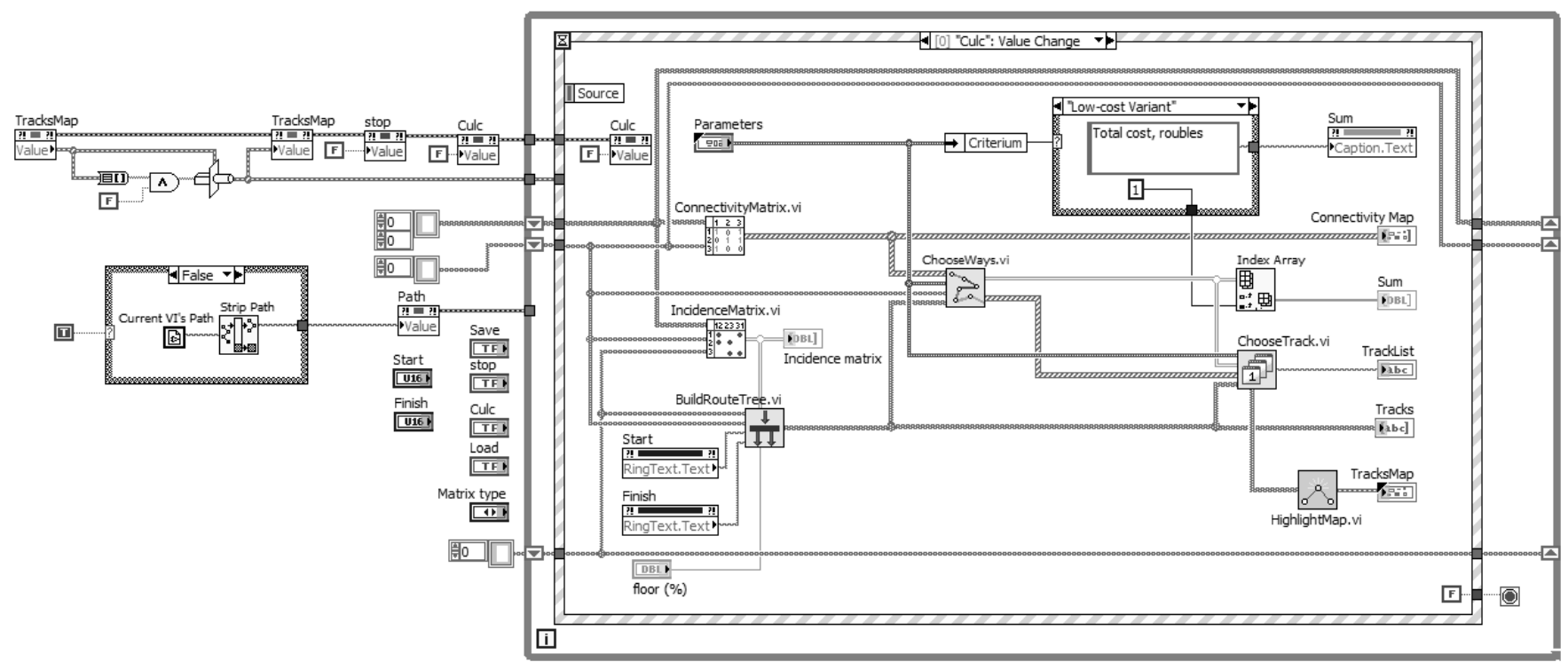

Fig. 5. Automatic forming of the route table in a program designed in LabVIEW language. Top teft panel - graphical user interface; top right panel menu page with the results of selecting the optimum route; bottom panel - block diagram of the main program. 


\section{CONCLUSION AND DISCUSSION OF RESULTS}

The suggested algorithm for dynamic forming of route tables allows one to find all available variants passenger transportations from the departure place to the destination point. Adjusting of the routing table-filling threshold can detect routes with minimum number of transitions. Selecting of transport modes and determining the optimum route are realized with taking into account the most important for passengers' criteria: total cost, journey time and comfort level.

Testing the software implementation of the algorithm by the example of Irkutsk Region tourist routs has shown its efficiency. Using string formats for storing variables in the program allows solving the routing task for arbitrary given names of route map points. Visual user interface of the program is very useful and convenient. It is also available to insert a map into the front panel of the program and illuminate routes with virtual light emitting diodes.

\section{References}

[1] T. Brands, E.C. van Berkum, "Performance of a Genetic Algorithm for Solving the Multi-Objective, Multimodal Transportation Network Design Problem," International Journal of Transportation, vol. 2, no. 1, pp. 1-20, 2014.

[2] C.E. Ferrell, M. Carroll, B. Appleyard, D. Reinke, S. Ashiabor, R. Dowling, H.S. Levinson, E. Deakin, and R. Cervero, Reinventing the Urban Interstate: A New Paradigm for Multimodal Corridors. Washington, DC: The National Academies Press, 2011.

[3] E.A. Kravchenko, A.E. Kravchenko, "Competitiveness Different Modes of Transport in the Resort Area Based on the Theory of Quality Logistics Transport Service of Bus Transport," International Journal of Experimental Education, vol. 1, no. 8, pp. 28-34, 2015.

[4] E. Martynova, S. Gusev, "Multimodal Freight and Passengers: Logistics Organization of Options," Modern Problems Of Russian Transport Complex, vol. 2, no. 3, pp. 145-153, 2013.

[5] A.S. Ermakov, "Autotourism Itinerary Development Methodology," Universities for Tourism and Service Association Bulletin, vol. 7, no. 3, pp. 39-44, 2014.

[6] M.S. Pozidaev, Algorithms for solving the transport problem of routing. Tomsk: Tomsk State University, 2010 [Ph.D. dissertation, p. 115, 2010].

[7] W. Fan, R. Machemehl, "Optimal Transit Route Network Design Problem with Variable Trans-it Demand: Genetic Algorithm Approach," Journal of Transportation Engieenring, vol. 132, no.1, pp. 40-51, January 2006.

[8] V.S. Tarasyan, A.Y. Polushkin, "Transportation Problem with a Mediatorand Its Solution Using Evolutionary Methods," Innotrans, vol. 17, no. 3, pp. 13-17, September 2015.

[9] A. Levitin, Introduction ot the Design \& Analysis of Algorithms, 1st ed. Villanova University: Addison-Wesley, 2003.

[10] E.W. Dijkstra, "A note on two problems in connexion with graphs," Numerische Mathematik, vol. 1, issue 1, pp. 269-271, December 1959.

[11] N.V. Krol, N.V. Davydova, R.Y. Upyr, "Organization of multimodal passenger transportation as the basis of touristic business in Eastern Siberia," Proceedings of International scientific and practical conference Transport-2015, vol. 1, pp. 135-137, April 2015. [Conf. Transport-2015, p. 505, 2015]. 